\title{
Chemically consistent evolution of galaxies
}

\section{Spectrophotometric evolution from zero to high redshift}

\author{
J. Bicker, U. Fritze-v. Alvensleben ${ }^{1}$, C. S. Möller ${ }^{2}$, and K. J. Fricke ${ }^{1}$ \\ 1 Universitätssternwarte, Geismarlandstraße 11, 37083 Göttingen, Germany \\ 2 Max Planck Institute for Astrophysics, Karl-Schwarzschild-Str. 1, 85741 Garching, Germany
}

Received 7 May 2003 / Accepted 15 September 2003

\begin{abstract}
The composite stellar populations of galaxies comprise stars of a wide range of metallicities. Subsolar metallicities become increasingly important, both in the local universe when going from early towards later galaxy types as well as for dwarf galaxies and for all types of galaxies towards higher redshifts.

We present a new generation of chemically consistent evolutionary synthesis models for galaxies of various spectral types from $\mathrm{E}$ through Sd. The models follow the chemical enrichment of the ISM and take into account the increasing initial metallicity of successive stellar generations using recently published metallicity dependent stellar evolutionary isochrones, spectra and yields.

Our first set of closed-box 1-zone models does not include any spatial resolution or dynamics. For a Salpeter initial mass function (IMF) the star formation rate (SFR) and its time evolution are shown to successfully parameterise spectral galaxy types $\mathrm{E}, \ldots, \mathrm{Sd}$. We show how the stellar metallicity distribution in various galaxy types build up with time to yield after $\sim 12 \mathrm{Gyr}$ agreement with stellar metallicity distributions observed in our and other local galaxies.

The models give integrated galaxy spectra over a wide wavelength range $(90.9 \AA-160 \mu \mathrm{m})$, which for ages of $\sim 12$ Gyr are in good agreement not only with observed broad band colours but also with template spectra for the respective galaxy types.

Using filter functions for Johnson-Cousins $U, B, V, R_{\mathrm{C}}, I_{\mathrm{C}}$, as well as for HST broad band filters in the optical and Bessel \& Brett's NIR $J, H, K$ filter system, we calculate the luminosity and colour evolution of model galaxies over a Hubble time. Including a standard cosmological model $\left(H_{0}=65, \Omega_{0}=0.1\right)$ and the attenuation by intergalactic hydrogen we present evolutionary and cosmological corrections as well as apparent luminosities in various filters over the redshift range from $z \sim 5$ to the present for our galaxy types and compare to earlier models using single (=solar) metallicity input physics only. We also resent a first comparison of our cc models to HDF data. A more detailed comparison with Hubble Deep Field (HDF) and other deep field data and an analysis and interpretation of high redshift galaxies in terms of ages, metallicities, star formation histories and, galaxy types will be the subject of a forthcoming paper.
\end{abstract}

Key words. galaxies: evolution - galaxies: stellar content - galaxies: photometry - galaxies: spectra - galaxies: redshifts cosmology: observations

\section{Introduction}

The number of high and very high redshift galaxies is increasing rapidly these days. Thousands of $U$ - and $B$-dropout galaxies with photometric redshift estimates are known from the HST Hubble Deep Fields - North and South (Williams et al. 1996, 1998; Gardner et al. 2000; Ferguson et al. 2000) - and from ground-based deep surveys like the VLT- Fors Deep Field (FDF) and others. Hundreds of these have spectroscopically confirmed redshifts up to $z \sim 6$ (e.g. Hu et al. 1999). Deep surveys are being conducted at all wavelengths from UV through IR and far into the sub-mm range. These brilliant data require mature galaxy evolution models for adequate interpretation. Ideally, these models should cover all the observational wavelength baseline to allow for a consistent

Send offprint requests to: J. Bicker, e-mail: jbicker@uni-sw.gwdg.de interpretation of all the available data, be as comprehensive and realistic as possible, and extend from very early phases at very high redshift towards the global properties of nearby galaxies of various spectral types. Moreover, an ideal galaxy model should be as simple as possible, involving the smallest possible number of free parameters. A comprehensive galaxy model should describe the evolution of as many observable quantities as possible (spectrum, luminosities, colours, emission and absorption features for the stellar population, the gas content and a large number of element abundances for the interstellar medium (ISM)). A realistic galaxy evolution model should consistently take into account both the age and metallicity distributions of the stellar populations that naturally result from any extended star formation history (SFH).

This is what we attempt with our chemically consistent spectrophotometric, chemical and cosmological evolutionary synthesis model. The chemical evolution aspects of this 
model were presented by Lindner et al. (1999), based on earlier less complete stellar evolutionary tracks, in comparison with and interpretation of the observed redshift evolution of Damped Ly $\alpha$ Absorber (DLA) abundances. Here, we present the spectral and spectrophotometric aspects of our unified chemical and spectral evolutionary synthesis code including current stellar evolutionary isochrones.

Spectrophotometric and cosmological evolutionary synthesis models generally applied in current interpretations of high redshift galaxy data are using solar metallicity input physics only together with specific parametrisations for the star formation (SF) histories of various spectral types (e.g. Bruzual \& Charlot 1993; Bressan et al. 1994; Guiderdoni \& Rocca-Volmerange 1987, 1988; Fioc \& Rocca-Volmerange 1997; Poggianti 1997). Broad stellar metallicity distributions extending down to fairly low $[\mathrm{Fe} / \mathrm{H}]$ are observed in local galaxies of various types. We will show in how far the consideration of a realistic metallicity distribution among the stars will affect model predictions for high redshift galaxies.

The first attempts to account for non-solar abundances and their impact on the photometric evolution of galaxies go back to Arimoto \& Yoshii (1986). Einsel et al. (1995) used more recent and complete stellar evolutionary tracks and colour calibrations for initial stellar metallicities $10^{-4}$ to $4 \times 10^{-2}$ to describe in a chemically consistent way the photometric evolution of galaxy types E through Sd. In Möller et al. (1997) we introduced the concept of chemical consistency into the spectrophotometric evolution of galaxies using Kurucz's (1992) model atmosphere spectra for a range of stellar metallicities and investigated the time evolution of ISM metallicity and luminosity-weighted mean stellar metallicities in various wavelength bands. For models that well agree with observed template spectra (Kennicutt 1992) of various types (E, Sb, Sd) we gave decompositions of the total light emitted at wavelengths from $U$ through $K$ in terms of luminosity contributions from various metallicity subpopulations. This clearly showed the considerable widths of the metallicity distributions in all 3 galaxy types as well as the importance of luminosity contributions from stellar populations with subsolar metallicities. Recently Vázquez et al. (2003) presented their evolution synthesis code SPECTRAL in application to the irregular galaxy NGC 1560 that also accounts for the the simultaneous presence of stars of different metallicities. They use stellar evolutionary input of the Geneva group and describe the spectral evolution of NGC 1560 on the basis on chemical evolution models by Carigi et al. (1999).

This paper is organised as follows. In Sects. 2 and 3 we present our chemically consistent galaxy evolution model and the various pieces of input physics for different metallicities that it uses. Section 4 gives a comparison of our model colours and spectra with observations of nearby galaxies to show that - after a Hubble time - our models do reproduce the observed properties of typical local galaxy types E ... Sd. In Sect. 5 we present the results for our models $\mathrm{E}, \mathrm{Sb}, \mathrm{Sd}$ in terms of spectra at various ages, apparent magnitudes, evolutionary and cosmological corrections (including attenuation) in wavelength bands $U B V R_{\mathrm{c}} I_{\mathrm{c}} J H K$ and a series of HST broad band filters as a function of redshift. Results are presented for the redshift range
$0 \leq z \leq 4.8$ for a cosmological model $H_{0}=65, \Omega_{0}=0.1$, with a redshift of 5 assumed for galaxy formation in both cases.

We compare our chemically consistent models to models using solar metallicity input physics only and present a first comparison with HDF galaxies with spectroscopic redshifts in Sect. 6. Conclusions are given in Sect. 7. A further set of models which also includes the influence of dust in a chemically consistent way will be presented a separate paper (see Möller et al. 2001a,b for first results). A detailed interpretation of the HDF data with the models presented here will be the subject of a forthcoming paper.

\section{Metallicity observations in galaxies}

For quite some time, observational evidence has been accumulating for sometimes very broad metallicity distributions of stars in normal galaxies. Stars in the Milky Way disk and halo span a range of at least 4 orders of magnitude in metallicity $-4.2 \leq[\mathrm{Fe} / \mathrm{H}] \leq+0.3$. While some years ago, the focus was on super-solar metallicities e.g. in (the centres of) massive ellipticals, bulges, X-ray halos around ellipticals, and the hot intra cluster medium (ICM), by today, it is clear that the average metallicities in all those cases are subsolar. The sun, our reference star, stands out in metallicity among solar neighbourhood stars. For F, G, K dwarfs the $[\mathrm{Fe} / \mathrm{H}]$ distributions extend from -0.8 to +0.4 (Rocha-Pinto \& Maciel 1998), while B-stars show $\langle[\mathrm{O} / \mathrm{H}]\rangle=-0.31$ (Kilian-Montenbruck et al. 1994). When averaged over $1 R_{\mathrm{e}}$, with $R_{\mathrm{e}}=$ effective radius, line strength gradients in ellipticals indicate $\left\langle Z_{*}\right\rangle \sim(0.5-1) \cdot Z_{\odot}$ (Carollo \& Danziger 1994). For stars in the bulge of our Milky Way $\left\langle Z_{*}\right\rangle \sim(0.3-0.7) \cdot Z_{\odot}$ (e.g. McWilliam \& Rich 1994; Sadler et al. 1996; Ramirez et al. 2000), for the X-ray gas halos around elliptical galaxies ASCA observations give $0.1 \leq[\mathrm{Fe} / \mathrm{H}] \leq 0.7$ (e.g. Loewenstein 1999).

Characteristic HII region abundances (i.e. measured at $1 R_{\mathrm{e}}$ ), which give an upper limit to the average gas phase abundance, range from $Z \gtrsim Z_{\odot}$ for Sa spirals down to $<\frac{1}{2} Z_{\odot}$ for Sd galaxies (e.g. Oey \& Kennicutt 1993; Zaritsky et al. 1994; Ferguson et al. 1998; van Zee et al. 1998). Locally, dwarf irregular galaxies have metallicities in the range $(2-30) \% Z_{\odot}$ (e.g. Richer \& McCall 1995). The first spectra of Lyman break galaxies at redshifts $z \sim 3-4$ have shown that their metallicities, derived from stellar wind features, are considerably subsolar, sometimes even sub-SMC (Lowenthal et al. 1997; Trager et al. 1997; Pettini et al. 2000; Teplitz et al. 2000). Neutral gas in damped Ly $\alpha$ absorbers observed to $z>4$ shows abundances in the range $-3 \lesssim[\mathrm{Zn} / \mathrm{H}] \lesssim 0$ (e.g. Pettini et al. 1997, 1999; Lindner et al. 1999).

\section{Chemically consistent galaxy evolution models}

From a very principle point of view it is clear that in contrast to single burst stellar populations like star clusters, any galaxy with a star formation history extending over one to several Gyr, i.e. much longer than the lifetime of massive stars, will have a stellar population that is composite not only in age but also in metallicity - as confirmed by the observations cited above. This is what we intend to account for in our chemically consistent 
galaxy evolution models. The basic concept of our evolutionary synthesis model for galaxies has been described in detail by Fritze-v. Alvensleben \& Gerhard (1994), the extended version allowing for a chemically consistent $(=\mathbf{c c})$ modelling is described by Einsel et al. (1995) for the photometric evolution and in detail by Möller et al. (1997), Möller et al. (1999), Fritze-v. Alvensleben et al. (1999) for the spectral and spectrophotometric evolution and by Lindner et al. (1999) for the chemical evolution.

In the following we briefly outline the principle of the new concept of chemical consistency which we consider an important step towards a more realistic galaxy modelling. In contrast to single burst single metallicity stellar populations like star clusters (Schulz et al. 2002) our chemically consistent galaxy evolution model, solving a modified set of Tinsley's equations with metallicity dependent stellar yields, follows the metal enrichment of the ISM and accounts for the increasing initial metallicity of successive stellar generations, both with respect to the evolution of ISM abundances (Lindner et al. 1999) and to the spectral evolution as presented here. The evolution of each star is followed in the HR diagram from birth to its final phases according to stellar evolutionary tracks appropriate for its initial metallicity so that at each timestep the distribution of all stars over the HRD is known. The evolution of the HRD population is followed with various sets of stellar isochrones from the Padova group for five different metallicities from $Z=4 \times 10^{-4}$ to $5 \times 10^{-2}$. Stellar subpopulations formed with initial metallicities in between two of the 5 discrete metallicities of the Padova isochrones are described by two components from the two adjacent metallicities. The relative contributions of these two components are weighted by the inverse of the logarithmic differences between the metallicity of the subpopulation and the metallicities of the adjacent isochrones. At any timestep the HRD population is used to synthesise an integrated galaxy spectrum from a library of stellar spectra. This library comprises stellar model atmosphere spectra from UV to the IR for all spectral types and luminosity classes for 5 metallicities (Lejeune et al. 1997, 1998). The total galaxy spectrum is obtained by summing the isochrone spectra, weighted by the star formation rate at birth of the stars on the respective isochrone for each metallicity and, finally, by coadding the spectra of the various single metallicity subpopulations. Combining the spectrophotometric time evolution with a cosmological model and some assumed redshift of galaxy formation we calculate the evolutionary and cosmological corrections as well as the evolution of apparent magnitudes from optical to NIR for various galaxy types taking into account the attenuation of the emitted galaxy light by intervening HI (cf. Sect. 3.4).

\subsection{Input physics}

In an attempt to keep the number of free parameters as small as possible, our models are calculated as closed boxes with instantaneous and perfect mixing of the gas. We use isochrones from stellar evolutionary tracks provided by Bertelli et al. (1994) (Padova group) in the version from November 1999 that include the thermal pulsing AGB phase as described in Schulz et al. (2002). The stellar lifetimes for the various metallicities are fully taken into account in our description of the ISM enrichment.

The only stellar library covering the whole range of stellar metallicities, spectral types, and luminosity classes is the library of model atmospheres by Lejeune et al. (1997, 1998), based on the original library of Kurucz (1992). Its wide wavelength range from 0.09 to $160000 \mathrm{~nm}$ extending far into the UV allows us to calculate cosmological corrections even in the $U$ band out to very high redshift $z \sim 5$. For stars hotter than $50000 \mathrm{~K}$, the highest effective temperature of Lejeune et al.'s library, we use black body spectra. To follow in detail the chemical evolution of the ISM, Lindner et al. (1999) included stellar yields from Woosley \& Weaver (1995) and van den Hoek \& Groenewegen (1997) for various metallicities. For the models in this paper we are not interested in the detailed chemical evolution. We only use the time evolution of the global metallicity $Z$ to know when to switch from one isochrone to the next (=more metal rich).

With this new set of metallicity dependent input physics our models are now chemically consistent both with respect to the spectrophotometric and to the chemical evolution.

The various spectral galaxy types of the Hubble Sequence of normal galaxies are described by their respective appropriate galaxy-averaged star formation histories. For spheroidal galaxies (E) models use a Star Formation Rate SFR $\sim \mathrm{e}^{-\frac{t}{t *}}$ with an $e$-folding time $t_{*}=1 \mathrm{Gyr}$. Following Kennicutt (1998) we assume for the spirals a SFR linearly proportional to the gas-tototal mass ratio with characteristic timescales for the transformation of gas into stars ranging from $t_{*}=4 \mathrm{Gyr}$ for Sa through $t_{*}>15 \mathrm{Gyr}$ for Sd spectral types.

We use a standard Salpeter IMF from lower to upper mass limits $m_{l}=0.08 M_{\odot}$ to $m_{\mathrm{u}} \sim 70 M_{\odot}$, as given by the isochrones. The IMF is normalised to a fraction of visible mass of $\mathrm{FVM}=0.5$ to match the mass-to-light $(\mathrm{M} / \mathrm{L})$ ratios for today's ( 12 Gyr old) galaxies.

A Scalo (1986) or a Kroupa (1993) IMF would produce a lower number of massive stars than the Salpeter IMF. Lindner et al. (1999) have shown that the metallicity evolution of spirals is too slow for a Scalo IMF as compared to observations. As compared to the Salpeter IMF that we use, these other IMFs, whithout adjustment of the SFRs, would produce a larger fraction of low metallicity stars and, hence, lead to slightly bluer galaxy colours.

\subsection{Filters and calibrations}

We calculate the apparent magnitudes $m_{\lambda}$, evolutionary and cosmological - corrections for the $U B V-$ Johnson \& $R_{\mathrm{C}} I_{\mathrm{C}}$ Cousins filter system which is taken from Lamla (1982), for the JHK filters given by Bessel \& Brett (1988), and for all broad band HST WFPC2 filters. Magnitudes in all filters are calibrated in the VEGAMAG system. On the basis of the time evolution of the model spectra we provide the evolution in other filter systems, easily calculated by directly folding the filter and detector response curves with the model spectra. 


\subsection{Cosmological model}

In order to compare our models with data of high redshift galaxies, we transform the spectrophotometric time evolution into a redshift evolution with a set of cosmological parameters $\left(H_{0}, \Omega_{0}, z_{\mathrm{f}}\right)$, where $z_{\mathrm{f}}$ is the redshift of galaxy formation.

The age of a galaxy at redshift $z=0$ is given by

$t_{0}:=t_{\mathrm{gal}}(z=0):=t_{\text {Hubble }}(z=0)-t_{\text {Hubble }}\left(z_{\mathrm{f}}\right)$.

After $t_{\mathrm{gal}}(z=0)$ models are normalised to the observed average absolute luminosities $M_{B}^{*}$ of the respective galaxy types in Virgo (cf. Sandage et al. 1985a,b). To obtain the observable apparent magnitudes $m_{\lambda}$ from the absolute magnitudes $M_{\lambda}$ given by our models, we calculate the evolutionary $\left(e_{\lambda}\right)$ and cosmological corrections $\left(k_{\lambda}\right)$ and the bolometric distance modulus $\operatorname{BDM}\left(H_{0}, \Omega_{0}\right)$ :

$m_{\lambda}(z)=M_{\lambda}\left(z=0, t_{0}\right)+B D M(z)+e_{\lambda}(z)+k_{\lambda}(z)$.

The cosmological or $k$-correction $k_{\lambda}$ in any wavelength band $\lambda$ describes the effect of the expanding universe, which redshifts a galaxy spectrum of local age $t_{0}$ to some redshift $z$.

$k_{\lambda}(z):=M_{\lambda}\left(z, t_{0}\right)-M_{\lambda}\left(0, t_{0}\right)$.

$k_{\lambda}$ can also be calculated from observed galaxy spectra. In this case, the maximum redshift to which this is possible depends on how far into the UV the observed spectrum extends. Our model galaxy spectra at $z=0$ extend from $90 \AA$ through $160 \mu \mathrm{m}$ and, hence, allow for cosmological corrections in optical bands up to $z \gg 10$.

The difference in absolute luminosities between two galaxies at the same redshift but with different ages is described by the evolutionary correction

$e_{\lambda}(z):=M_{\lambda}\left(z, t_{\mathrm{gal}}(z)\right)-M_{\lambda}\left(z, t_{0}\right)$

Evolutionary corrections, of course, cannot be given without an evolutionary synthesis model. It will be shown in Sect. 5 for which galaxy types at which redshifts evolutionary corrections become important.

It is important to stress that both the evolutionary and the cosmological corrections do not only depend on the cosmological parameters but also on the SFH, i.e. on the spectral type of the galaxy.

In this paper we present cosmological and evolutionary corrections and apparent magnitudes for cosmological parameters $\left(H_{0}, \Omega_{0}\right)=(65,0.1)$ and the formation of galaxies at redshift $z_{\mathrm{f}}=5$. This cosmology gives a galaxy age of about $12 \mathrm{Gyr}$ after a Hubble time and, hence, makes sure that the agreement with colours and spectra of nearby galaxies at $z=0$ is given. Any combination of $H_{0}$ and $\Omega_{0}$ which gives a local galaxy age $t_{\text {gal }} \lesssim 10$ Gyr can be excluded because the very red colours of ellipticals can only be reached after $\gtrsim 12$ Gyr and globular cluster ages also are of order 12 to 15 Gyr. In particular, high values of $H_{0}>80$, even in combination with low values for $\Omega_{0}$, yield galaxy ages less than $10 \mathrm{Gyr}$.

\subsection{Attenuation}

For very distant galaxies the cumulative effect of neutral hydrogen stochastically distributed along our lines of sight, mostly in the form of Ly $\alpha$ clouds, significantly attenuates the emitted light at wavelengths shorter than rest-frame Ly $\alpha$ at $1216 \AA$. From his analysis of a large number of lines of sight to distant and very distant quasars, Madau (1995) derived a statistically averaged attenuation correction of the form $\operatorname{att}(\lambda, z)$ which we include into the cosmological corrections of our models. The effect of attenuation becomes visible in $U$ at $z \gtrsim 2$, in $B$ at $z \gtrsim 2.5$, in $V$ at $z \gtrsim 3.5$, etc.

\subsection{Discussion of models}

Our models describe the global spectrophotometric evolution of field galaxies. In particular, our E model represents the classical model for a normal elliptical galaxy with average luminosity and metallicity. A hierarchical or major merger origin of ellipticals is not investigated here. The closed-boxes we assume for our spiral models clearly are a poor approximation only motivated by our intend to keep this first models as simple as possible an the number of free parameters to a minimum. For a hierarchically accreting spiral the total SFH of its ensemble of subclumps on reasonable time averages should not differ mutch from what our simplified models assume in order to get the correct colours and spectra at $t_{0}$. The same had been to be true for the chemical evolution of an ensemble of subclumps as compared to our simplified closed-box models by Lindner et al. (1991). The colour evolution hence is not significant affected by our simplification and, as far back as the mass of a realistic accreting galaxy is of order $\geq 30 \%$ of its mass today the differences in apparent luminosities can be estimated to be smaller than differences between different galaxy types. In a previous paper we have shown that our models well describe the stellar metallicity as observed by means of absorption indices (Möller et al. 1997) and the chemical evolution of nearby and high redshift spiral galaxies (Lindner et al. 1999).

In this paper we restrict ourselves to models without dust. The effect of dust absorption is analysed in Möller et al. (2001) with our old track based models. A set of models including dust will be presented by Möller et al. (in prep.).

\section{Comparison with nearby galaxies}

\subsection{Colours}

Star formation histories for our model galaxies are chosen such that after a Hubble time or, more precisely, after the evolution time of a galaxy from its formation at redshift $z_{\mathrm{f}}$ to the present at $z=0$ as given by the cosmological model, the colours of our model galaxies agree with those observed for nearby galaxies of the respective type by Buta et al. (1995), e.g. $(B-V)=0.92,0.61$, and 0.50 for $\mathrm{E}, \mathrm{Sb}$ and $\mathrm{Sd}$ spectral types, respectively. As a consequence, however, our $V-K$ colours are then bluer by up to $0.3 \mathrm{mag}$ for Es and by up to $0.7 \mathrm{mag}$ for late-type spirals as compared to the observations of Aaronson (1978). This is partly due to the fact that our models describe the integrated colour of the entire stellar population, while the 
observations refer to the inner parts of the galaxies. Taking into account typical observed gradients in $V-K$ (cf. Fioc \& Rocca-Volmerange 1999) brings our $V-K$ colours into better agreement with observations, but there still remains a difference of about $0.5 \mathrm{mag}$ for late-type spirals. As we will show in Möller et al. (in prep.), the inclusion of reasonable amounts of dust will bring models into very good agreement with observations over the entire wavelength range from UV through $K$.

\subsection{Spectra}

In Fig. 1 we compare our model spectra with templates from Kennicutt's (1992) atlas for various spectral types. Note the very good agreement between our models $\mathrm{E}, \mathrm{Sa}, \mathrm{Sb}, \mathrm{Sc}$ with ages of about 12 Gyr with the observed spectra of NGC 4889 (E4), NGC 3471 (Sa), NGC 1832 (SBb), and NGC 6643 (Sc). The spectrum of NGC 4449 (Sd) is best modelled by our $\mathrm{Sd}$ galaxy with an age of about 4 Gyr in agreement with results found by Bruzual et al. (1993).

We like to point out that the spectral differences among galaxies of the same type in Kennicutt's library are larger than the differences between the template galaxies and our models.

Note that we have not included the gaseous emission-lines or continuum-lines in our models yet. In a next step, gaseous lines and continuum emission will be included in our cc models of actively star forming galaxies on the basis of metallicity dependent Lyman continuous fluxes and line ratios as already shown for single burst single metallicity models by Anders et al. (2003).

The complete set of galaxy model spectra for the various spectral types and ages between 4 Myr to 15 Gyr are given in machine readable tables and can also be found on our homepage http://www. uni-sw.gwdg.de/ galev. In Fig. 2 we show the time evolution of model spectra, on the example of an Sa model at 1, 3, 6, and 12 Gyr.

\subsection{Metallicities}

Our code follows the evolution of both the average ISM metallicity and the luminosity-weighted metallicities of the stellar population as seen in different bands. For a galaxy age of $12 \mathrm{Gyr}$, the average ISM metallicity is $\left\langle Z_{\mathrm{ISM}}\right\rangle=Z_{\odot}$ for E galaxies, $\left\langle Z_{\mathrm{ISM}}\right\rangle=1.5 \cdot Z_{\odot}$ for $\mathrm{Sa},\left\langle Z_{\mathrm{ISM}}\right\rangle=0.8 \cdot Z_{\odot}$ for $\mathrm{Sb}$, $\left\langle Z_{\text {ISM }}\right\rangle=0.5 \cdot Z_{\odot}$ for $\mathrm{Sc}$, and $\left\langle Z_{\mathrm{ISM}}\right\rangle=0.25 \cdot Z_{\odot}$ for $\mathrm{Sd}$ spirals, respectively. These values are in good agreement with observations of the characteristic (=measured at $\sim 1 R_{\text {eff }}$ ) HII region abundances in the respective spiral types (e.g. Oey \& Kennicutt 1993; Zaritsky et al. 1994; Phillips \& Edmunds 1996; Ferguson et al. 1998; van Zee et al. 1998). (See also Sect. 2.)

Depending on the SFH of the galaxy, the average stellar metallicity may differ by various degrees from the ISM metallicity and may also be different in different wavelength regions where stars of various masses, ages, and hence metallicities dominate the light (cf. Möller et al. 1996).

In Fig. 3 we show the relative luminosity contributions to $U, V$, and $K$ bands from stars of our 5 different metallicity subpopulations for $12 \mathrm{Gyr}$ old $\mathrm{E}$ and $\mathrm{Sb}$ models. For each
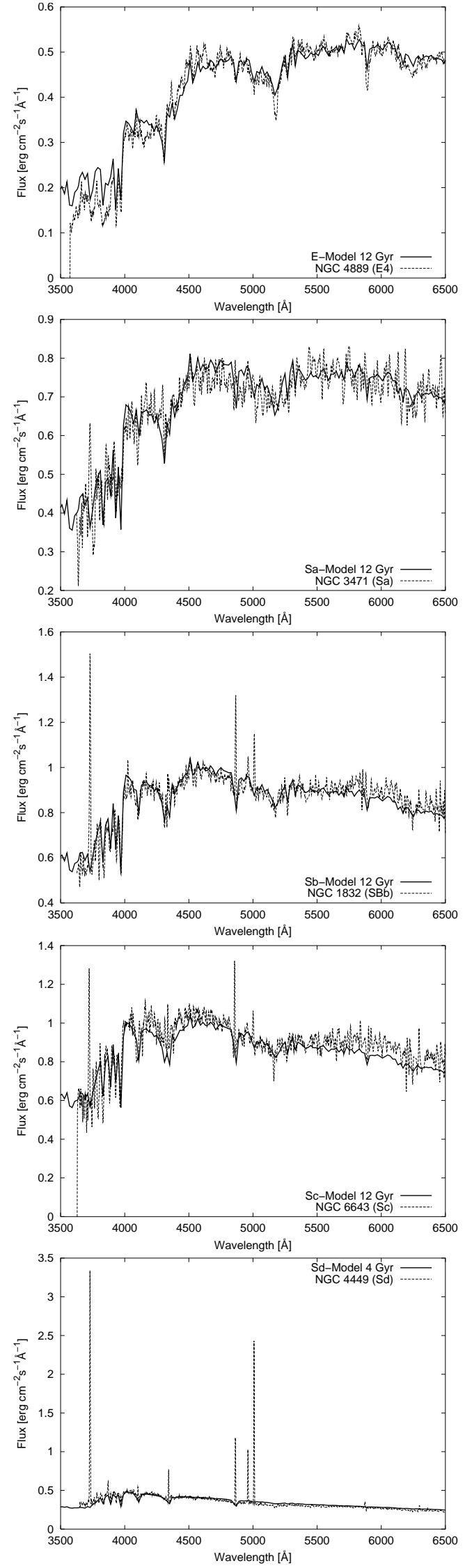

Fig. 1. Comparison of our model spectra (solid) at an age $T_{\text {gal }} \sim$ $12 \mathrm{Gyr}(\mathrm{E}, \mathrm{Sa}, \mathrm{Sb}, \mathrm{Sc})$ and $T_{\text {gal }} \sim 4 \mathrm{Gyr}(\mathrm{Sd})$ with Kennicutt's template spectra (dotted). 


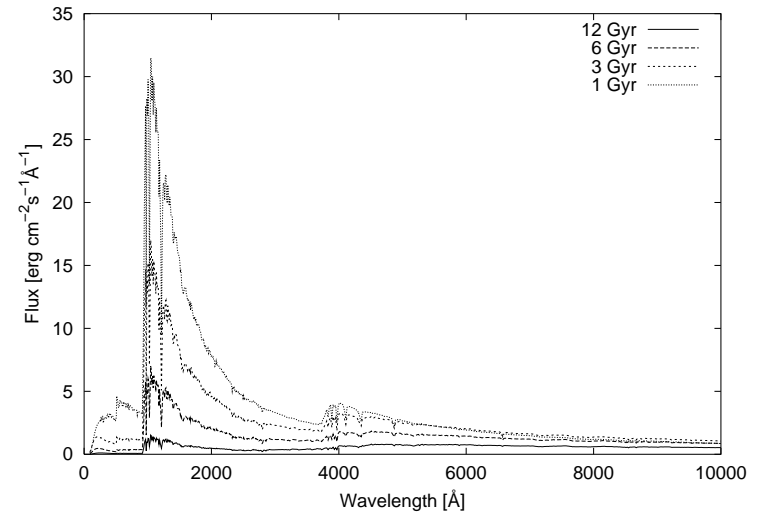

Fig. 2. Time evolution of Sa model spectra for ages 1, 3, 6 and $12 \mathrm{Gyr}$ (bottom to top). Fluxes are in units of erg cm $\mathrm{cm}^{-2} \mathrm{~s}^{-1} \AA^{-1}$, wavelength in $\AA$.
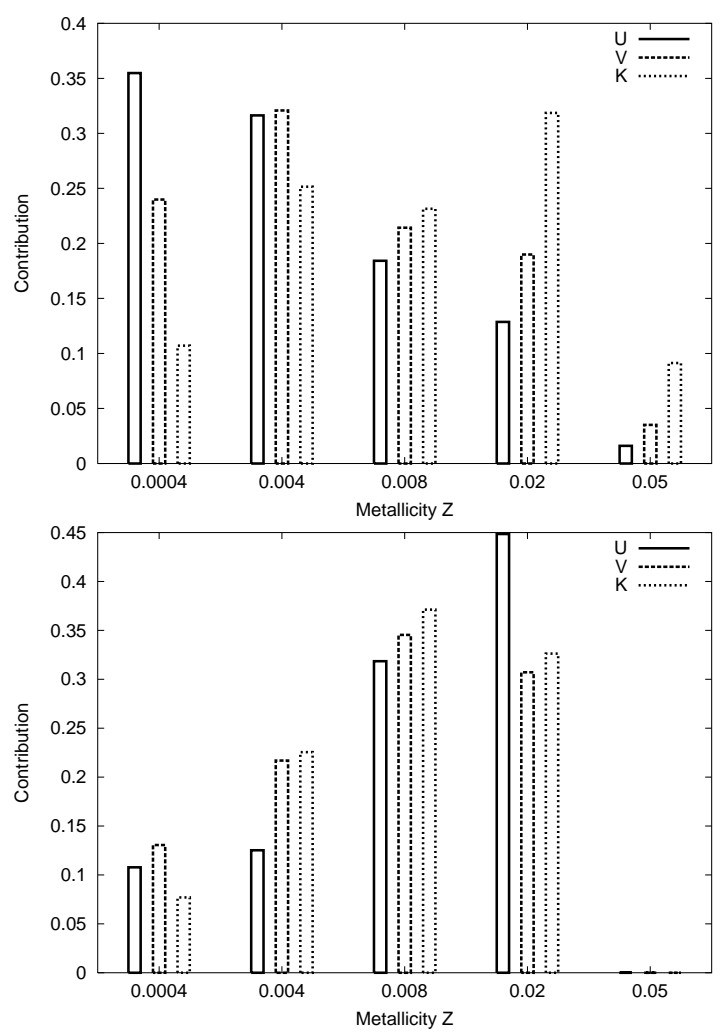

Fig. 3. Relative luminosity contributions to $U, V, K$ bands from stellar subpopulations of various metallicity for $12 \mathrm{Gyr}$ old $\mathrm{E}$ (top) and $\mathrm{Sb}$ (bottom) model.

wavelength band, the sum of the contributions from the different metallicities adds up to $100 \%$. Note the broad stellar metallicity distribution of the E model extending from $Z=4 \times 10^{-4}$ to $Z=0.05$ in good agreement with observed stellar metallicity distributions in resolved nearby ellipticals and bulges (cf. McWilliam \& Rich 1994).

The distribution differs from band to band. E.g., stars with low metallicity, e.g. $Z=0.0004$, contribute about 3 times more light to the $U$ - than to the $K$-band, while these relative contributions are reversed for stars of higher metallicity.

For models with different SFHs the stellar metallicity distributions are, of course, quite different. For stars in our global

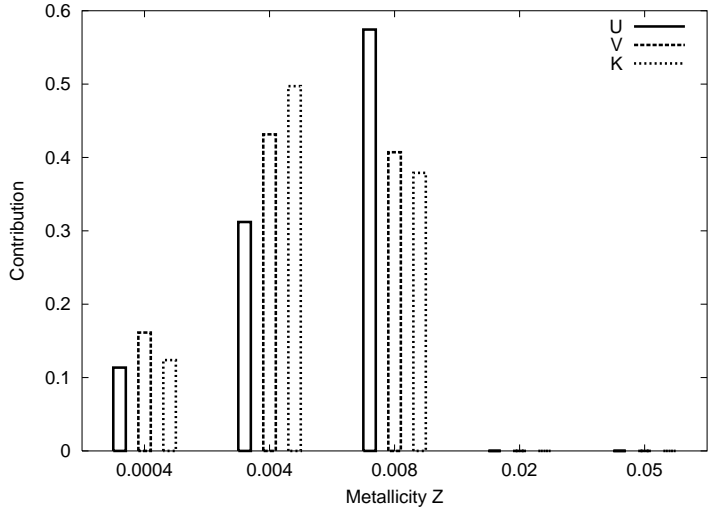

Fig. 4. Relative luminosity contributions to $U, V, K$ bands from stellar subpopulations of various metallicity for a $6 \mathrm{Gyr}$ old $\mathrm{Sb}$ model.

Sb model e.g., the metallicity distribution does not extend beyond $\sim Z_{\odot}$, and the differences between the relative contributions of a subpopulation of given metallicity to different bands are smaller than in the E-model. For Sd models, the stellar metallicity distribution is sharply peaked at $Z \lesssim \frac{1}{2} Z_{\odot}$ with small differences only between the different wavelength bands and also to the ISM metallicity. This is readily understood as a consequence of the long star formation timescale in Sd galaxies (cf. Möller et al. 1997 for details).

Similar to Fig. 3b, Fig. 4 shows the relative luminosity contribution of stellar subpopulations of different metallicities to the light in $U, V$, and $K$ emitted by an Sb galaxy, now at a younger age of 6 Gyr only. By that age - corresponding to a redshift $z \sim 0.5$ in our cosmology - , no stars of solar metallicity were present in this type of galaxy.

While Figs. 3 and 4 give the luminosity contributions of different metallicity subpopulations at ages of 12 and 6 Gyr, the time evolution of the luminosity contributions of stellar subpopulations of different metallicities to the $V$-band is shown in Fig. 5 for $\mathrm{E}$ and $\mathrm{Sb}$ galaxy models.

It is seen that the broad stellar metallicity distribution of the $\mathrm{E}$ model is already established at very young ages despite the present-day small stellar age distribution. Note in particular that despite its SFR declining rapidly on the short timescale of $t_{*}=1 \mathrm{Gyr}$, our E model - due to its realistic stellar metallicity distribution - will differ significantly in its spectrophotometric evolution from that of any single metallicity single burst model often used in the literature for the interpretation of E galaxy observations (see also Vazdekis et al. 1996, 1997).

Over more than the last $50 \%$ of its lifetime, the $V$-band light of the elliptical model is coming from stellar subpopulations of 4 different metallicities ( $Z=0.0004-0.02)$ at roughly comparable rates. The $V$-light of the Sb-model is seen to have been dominated by stars with half-solar metallicity during the second half of its lifetime. Only very recently, i.e. at ages $\geq 10 \mathrm{Gyr}$, solar metallicity stars gained importance while at early stages $<6$ Gyr only stars with $Z \leq 1 / 4 Z_{\odot}$ where present.

Figure 5 very clearly shows that at earlier evolutionary times, as observed in galaxies at high redshift, stars of lower and lower metallicities become dominant in the spectra of all spiral galaxies, though not for the E-model. And this is, 

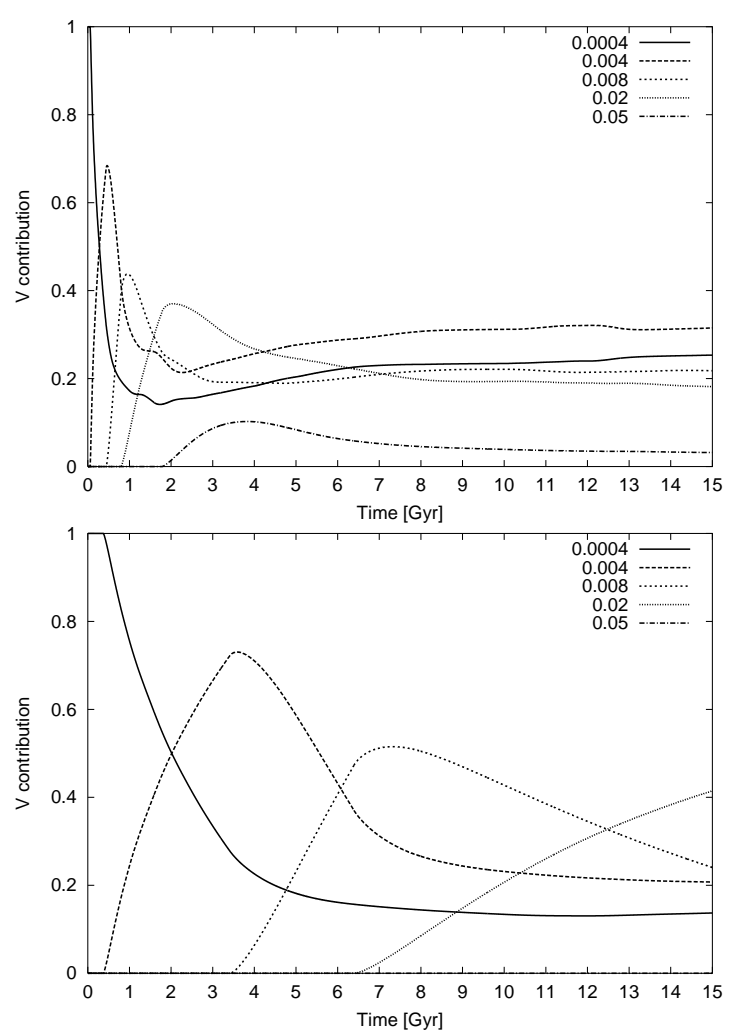

Fig. 5. Time evolution of the luminosity contribution to the $V$-band of the different metallicity subpopulations to the total luminosity for the E (top) and Sb (bottom) models.

of course, not only true in the $V$-band shown here, but over all wavelengths.

Figure 6 shows the time evolution of the luminosityweighted mean stellar metallicities, as defined in Möller et al. (1998), in different passbands for E, Sb, and Sd models. These luminosity weighted mean stellar metallicities in certain passbands are what metallicity dependent absorption features in the respective wavelength range of the integrated galaxy spectra are expected to measure. As already indicated in Fig. 3 for an age of 12 Gyr there is a significant difference in the luminosity weighted mean stellar metallicity in various bands in the E-model and this is seen in Fig. 6 to have persisted for all ages $\geq 7$ Gyr. The $K$-band shows the highest stellar metallicity of $\left\langle Z_{K}\right\rangle \sim 0.014$, more than $50 \%$ higher than that seen in $V:\left\langle Z_{V}\right\rangle \sim 0.008$. In the rest-frame $K$ the time evolution of the seen stellar metallicity is strongest. While it hat rapidly reached a maximum of $\left\langle Z_{K}\right\rangle_{\max } \sim Z_{\odot}$ at an age around $3 \mathrm{Gyr}$ it tremendously decreases to a present $\left\langle Z_{K}\right\rangle \sim 1 / 3 Z_{\odot}$ since then. The light emitted in rest-frame $U$ at a time around $3 \mathrm{Gyr}$, corresponding to a redshift $z \leq 1.5$ is shifted to observer-frame I, so we expect to see much higher metallicities in high- $z$ ellipticals than in local ones.

Due to the longer timescales of SF the metallicity differences between different passbands get much smaller towards later galaxy spectral types, as seen in Fig. 6, and their time evolution gets much slower and monotonic. Note the scale differences among E, Sb, and Sd models.
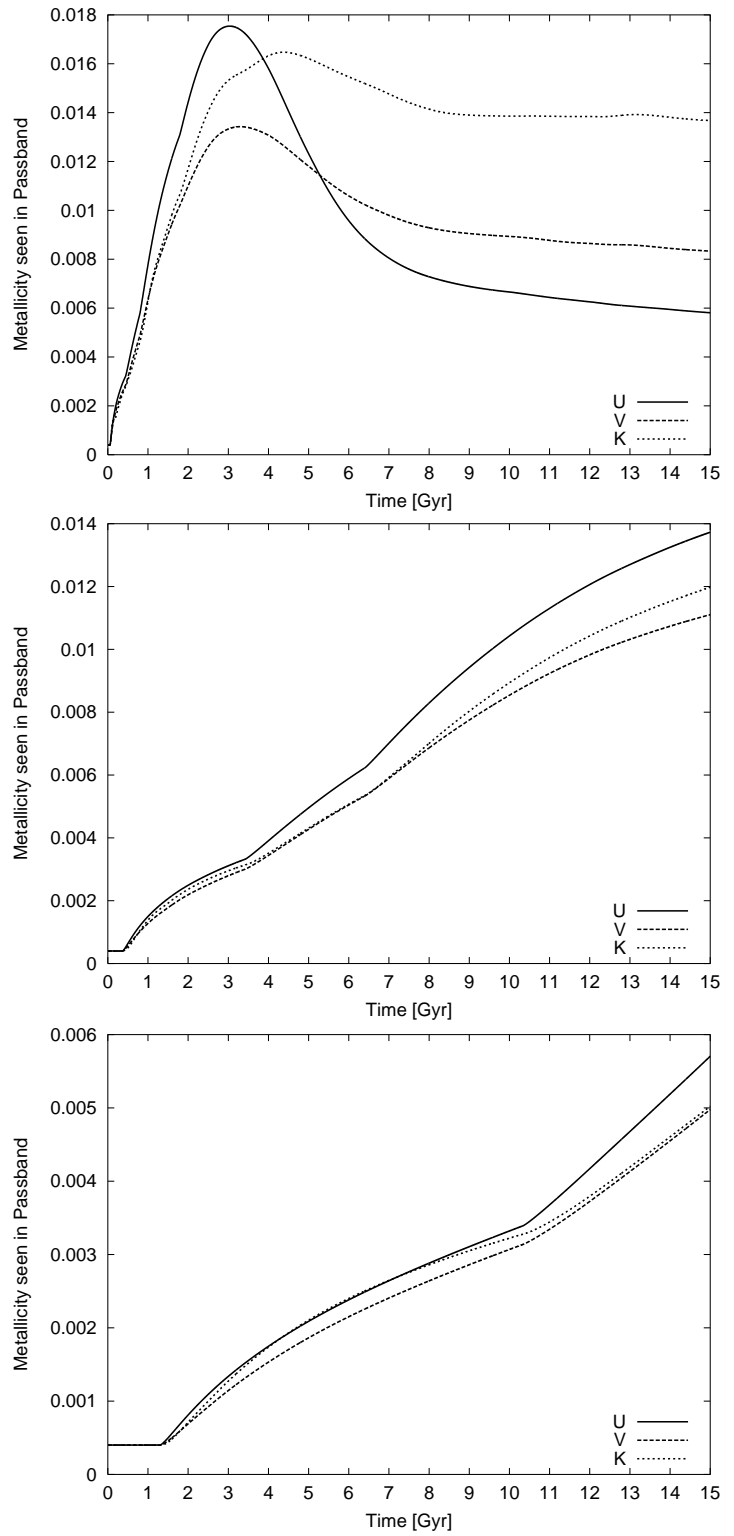

Fig. 6. Time evolution of the luminosity-weighted stellar metallicities in $U, V$, and $K$ (restframe) for different spectral types $\mathrm{E}, \mathrm{Sb}, \mathrm{Sd}$ (top to bottom).

\section{Evolutionary and cosmological corrections}

All evolutionary and cosmological corrections published so far were calculated with models using solar metallicity input physics only (e.g. Bruzual \& Charlot 1993; Poggianti 1997; Fioc et al. 1997). Also still widely in use are the $k$-corrections from Coleman et al. (1980) extracted from observed template spectra, although no evolutionary corrections are available in this case. The wavelength range of Colman et al. observation (1400-10000 $\AA$ ) limits the redshift range for their $k$-corrections to $z \lesssim 1$ in $U$ and to $z \lesssim 2$ in the $R$-band.

To cope with observations of very high redshift galaxies up to $z \sim 5$, models are needed that include the far UV as well as evolutionary corrections. 


\subsection{Comparison with solar metallicity models}

In the following we will focus on the differences as a function of redshift between our chemically consistent models (cc models) and models using solar metallicity input physics only ( $Z_{\odot}$ models). To isolate the effects of the chemically consistent treatment from those due to differences in the codes of various authors and/or the particular sources of input physics chosen (Padova vs. Geneva stellar evolutionary tracks, observed stellar spectra vs. model atmosphere libraries, etc.) we $\operatorname{ran} Z_{\odot}$ models with our code and the same solar metallicity input physics as in our cc models. The comparison between $\mathrm{cc}$ and $Z_{\odot}$ models is done for cosmological parameters $\left(H_{0}, \Omega_{0}\right)=(65,0.1)$. Note that the attenuation is not included in this comparison because it dominates the the $e+k$-corrections at high redshifts and would mash the metallicity effect.

In order to obtain agreement, after a Hubble time, with observed colours of the respective galaxy types (and, hence, with our cc model colours) the SFHs have to be slightly different in the models that do not contain the - on average - bluer and more luminous contributions of low metallicity stars. The $Z_{\odot}-E$ model hence is to be described by an exponentially decreasing SFR with an e-folding time of $t_{*} \sim 2 \mathrm{Gyr}$ as compared to $t_{*} \sim 1 \mathrm{Gyr}$ for the cc E model. The $Z_{\odot}-\mathrm{Sb}$ model needs $t_{*} \sim 9 \mathrm{Gyr}$ as compared to $t_{*} \sim 7 \mathrm{Gyr}$ for the cc Sb model. And $t_{*}>15 \mathrm{Gyr}$ for the $Z_{\odot}$ and the cc Sd model.

Figures 7 and 8 show a comparison of $e$ - and $k$-corrections in various bands as a function of redshift for the $\mathrm{cc}$ and $Z_{\odot}$ models. The most obvious difference between $\mathrm{cc}$ and $Z_{\odot}$ models is seen for the $\mathrm{E}$ model at short wavelengths. While for redshifts $z \lesssim 1.2$, the cc $(e+k)$ corrections in $U$ and $B$ are positive, making the apparent magnitudes of ellipticals fainter, those of the $Z_{\odot}$ model get increasingly negative from $z=0$ through $z \sim 2.5$ in $U$ and to $z \sim 3$ in $B$, respectively (cf. Fig. 7), making ellipticals apparently brighter

The maximum difference for the $\mathrm{E}$ models is as much as $\sim 3 \mathrm{mag}$ in $U$ and $\sim 2 \mathrm{mag}$ in the $B$-band at $z \sim 1$. Hence, cc models with a SFR decreasing exponentially with an e-folding time of 1 Gyr predict a smaller number of ellipticals to be expected at $z \lesssim 2.5$ in magnitude limited samples. At redshifts $\gtrsim 2.5$ the situation is reversed and the cc models appear more luminous. But our simple classic E model may not applicable at this redshift range. At longer wavelengths, e.g. $K$, differences are very small by redshift $\sim 1$ and increase to $\sim 1.5 \mathrm{mag}$ by $z=4$.

For $z \lesssim 2$ the differences in $U$ and $B$ between our $\mathrm{Sb}$ and $\mathrm{Sd} \mathrm{cc}$ and the respective $Z_{\odot}$ models are small. At the maximum the cc model has a $\sim 0.1$ mag higher correction. At redshifts higher than 2 the differences of the models increases and the $Z_{\odot}$ models have higher corrections than the cc models. The difference grow up to $1-1.5 \mathrm{mag}$ in the $U$ and $B$ band at high redshift. For the $K$-band the $Z_{\odot}$ models have always an higher $e+k$-correction than the cc models with a maximum difference of 0.4-0.5 mag at $z \sim 2$.

Hence, on the basis of our cc models we expect a slightly smaller number of late-type spirals by redshifts $z \lesssim 2$ to show up in magnitude limited surveys ( $\mathrm{U}$ and $\mathrm{B}$ ) as compared to $Z_{\odot}$
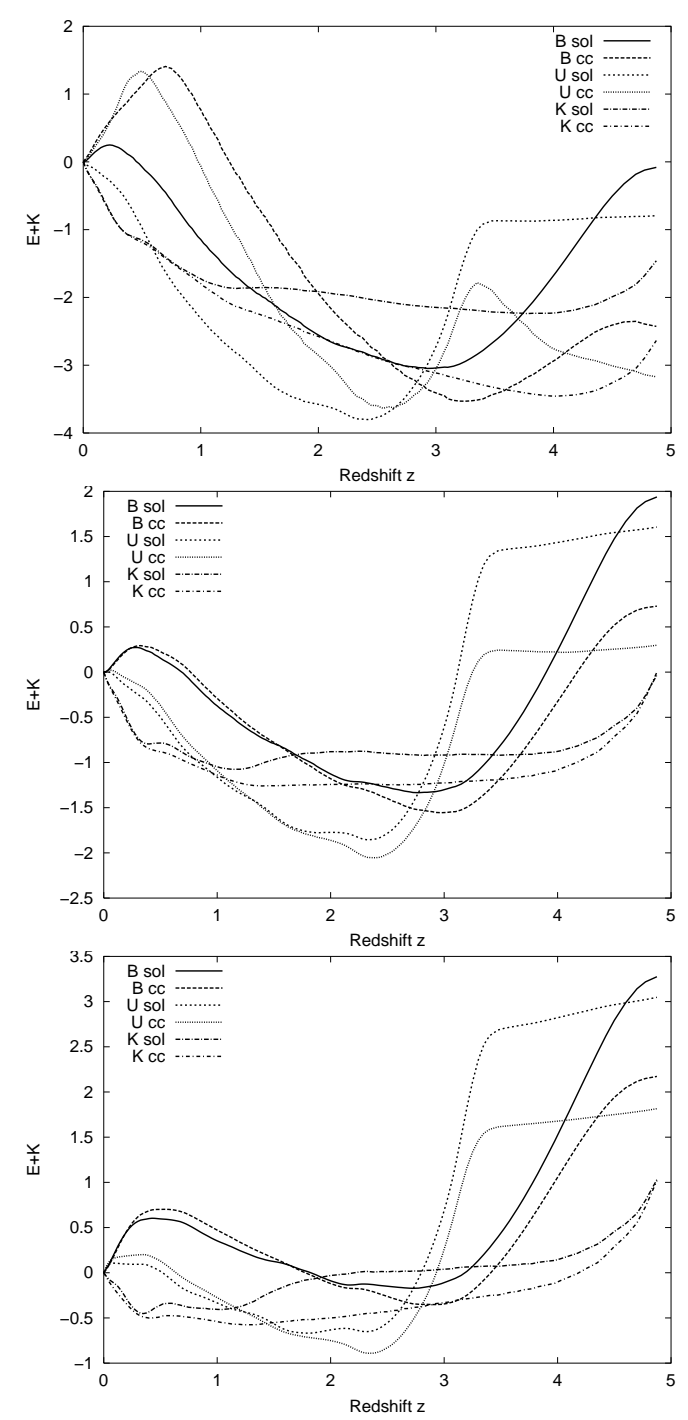

Fig. 7. Comparison of the $(e+k)$ corrections in $\mathrm{cc}$ and $Z_{\odot}$ models for $\mathrm{E}$ (top), $\mathrm{Sb}$ (middle) and $\mathrm{Sd}$ (bottom) galaxies in $U, B$ and $K$ band $\left(H_{0}, \Omega_{0}\right)=(65,0.1)$.

model predictions. At $z \gtrsim 2$ we expect more late-type spirals as compared to the $Z_{\odot}$ models

Figure 8 presents the decomposition of the $(e+k)$ corrections shown in Fig. 7 into the evolutionary and cosmological corrections $e_{\lambda}(z)$ and $k_{\lambda}(z)$ for the cc and $Z_{\odot}$ Sb model in the $U_{-}, B-$, and $K$-bands.

Let us first look at the $e$-correction. In the $Z_{\odot}$ model $B$ band $e$-corrections become increasingly negative from $z=0$ to $z \sim 2$ and remain constant around $e_{B} \sim-1.5$ mag all through $z>4$. In the cc model $e_{B}$ decreases faster to $e_{B} \sim-3$ at $z \sim 5$. Without the strong compensating effect of the $k$-correction the strongly negative $e_{B}$-correction would make the cc Sb model appear much brighter at $z \gtrsim 1.5$ than the $Z_{\odot}$ model. At $z \gtrsim 1.5$ the galaxies in our cosmology have ages $\$ 2.5 \mathrm{Gyr}$ and stars of low metallicities $Z \sim 0.0004-0.004$ dominate the light in $B$. The integrated spectrum still shows a high flux in $B$ so shortly after the major SF epoch. The behaviour of the $e_{U}$-correction is very similar to $e_{B}$. In $K$ the evolutionary corrections are $e_{K} \lesssim$ -0.5 through $z \sim 4$ for both, the cc and $Z_{\odot}$ Sb-models. 

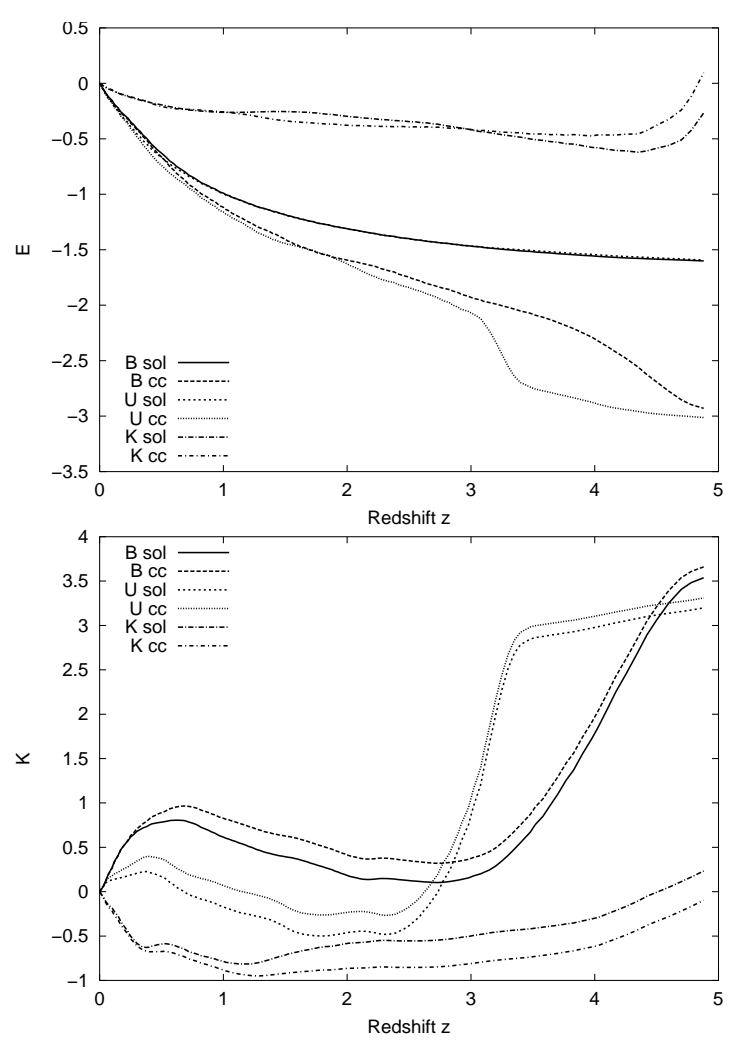

Fig. 8. Comparison of $\mathrm{cc}$ and $Z_{\odot}$ models for $\mathrm{Sb}$ in the $U$-, $B$-, and $K$-band. Decomposition into evolutionary (top) and cosmological (bottom) corrections.

For the $k$-corrections, the situation is quasi reversed. Till $z \sim 0.5$ the $k_{B}$-correction for the cc Sb model increases to $k_{B} \sim$ 1 and then it slightly falls to $k_{B} \sim 0.4$ at $z \sim 3$. Beyond $z=3$, $k_{B}$ increases rapidly to 3.7 at $z \sim 5$. For the $Z_{\odot}$ model $k_{B}$ follows a trend similar to that of the cc model but with an offset of $\sim-0.3$. The $k_{U}$-corrections show a redshift evolution similar to that of $k_{B}$. However, the $k_{U}$ is smaller than $k_{B}$ at lower redshifts $(\lessgtr 0.4)$ and negative in the redshift range $z \sim 1.3-2.6$. From $z \sim 2.6-3.5$ the $k_{U}$-correction increases from 0 to $\sim 3$ as the Lyman-break gets redshifted into the $U$-band. Beyond $z=3.5$ the Lyman-break is shifted out of the $U$ passband and hence the curve flattens. The difference between the cc and the $Z_{\odot}$ model is again an offset of $\sim-0.3$. The $K$-band $k$-correction for the cc model is always negative. It drops down to $k_{K} \sim-0.9$ at redshift $z \sim 1.3$ and rises to $k_{K} \sim 0$ at $z=5$. Until $z=1$ the difference between cc and $Z_{\odot}$ models is small $(\lessgtr 0.1)$. At redshifts $\gtrsim 1.5$ the cc model has an offset in $k_{K}$ of $\sim 0.3-0.4$.

The $k$-corrections and the $e+k$-corrections shown before do not include the effect of attenuation to highlight the differences between the $\mathrm{cc}$ and the $Z_{\odot}$ models. Figure 9 shows the $k$-corrections for the $\mathrm{Sb}$ model including the attenuation (cf. Fig. 8). It is seen that the attenuation is the dominant effect beyond $z=3$ in $U$ and $z=4$ in $B$.

\subsection{Comparison with earlier work}

We compare both our model $e$ - and $k$-corrections to those of Poggianti (1997). She calculated the $e$ and $k$ correction for

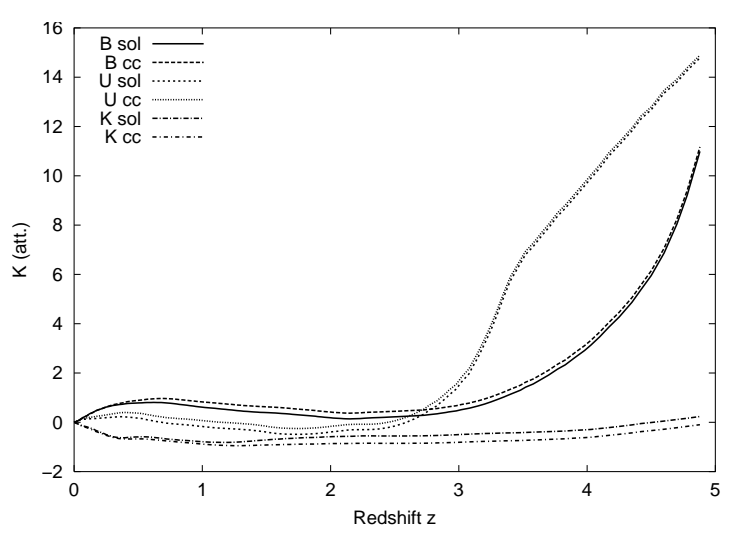

Fig. 9. $K$-correction - including the attenuation - for the Sb-model.

Table 1. Evolutionary and cosmological corrections for $\left(H_{0}, \Omega_{0}\right)=(65,0.1)$. The complete version of the table is given on our homepage.

\begin{tabular}{|r|rr|rr|l|rr|}
\hline \hline \multicolumn{7}{|c|}{ Sb model } \\
\hline$z_{r}$ & $e_{U}$ & $k_{U}$ & $e_{B}$ & $k_{B}$ & $\ldots$ & $e_{K}$ & $k_{K}$ \\
\hline 0.0 & 0.00 & 0.00 & 0.00 & 0.00 & $\ldots$ & 0.00 & 0.00 \\
0.1 & -0.17 & 0.18 & -0.16 & 0.26 & $\ldots$ & -0.05 & -0.22 \\
0.2 & -0.33 & 0.26 & -0.29 & 0.52 & $\ldots$ & 0.62 & -0.42 \\
0.3 & -0.48 & 0.34 & -0.41 & 0.71 & $\ldots$ & 0.52 & -0.61 \\
. &. & & & & $\ldots$ & & \\
$:$ & $:$ & & & & $\ldots$ & & \\
\hline
\end{tabular}

solar metallicity models. Note that her models do not include the attenuation by intergalactic $\mathrm{HI}$ and - due to a shorter wavelength coverage - do not allow for cosmological corrections in $U$ for $z>2$ and in $B$ for $z>2.5$. So we compare with our non attenuated cc models at this point. We also calculate the $e$-correction for the cosmology given by Poggianti (1997) for this comparison $\left(H_{0}=50, \Omega_{0}=0.5\right)$. Figures 10 and 11 show the comparison between our E and Sc cc models with the respective models form Poggianti (P) in the $U$ - and $B$-band. Note the good agreement of both models.

\section{Redshift evolution of apparent magnitudes}

The redshift evolution of apparent magnitudes in $U, B, V, R_{\mathrm{C}}, I_{\mathrm{C}}, J, H, K$ and HST filters is given on our homepage.

In Fig. 12, we show the redshift evolution of apparent magnitudes for the E, Sa, and Sd model in in HST $m 450$-band $(\sim$ Johnson $B)$ and $(m 450-m 606) \sim(B-V)$ colours and compare to the photometric redshift catalogue for the HDF galaxies (Sawicki et al. 1997). For each model the evolution is plotted with (thick lines) and without (thin lines) attenuation.

Before we present a very first and preliminary comparison of cc models with LBG data we recall that 1 st our models refer to integrated properties of galaxies and 2nd our model galaxy types - being described by SFHs or SF timescales - refer to a spectral and not to a morphological classification of galaxies. The question how far back in time the locally observed 1-to1 correspondence between spectral and morphological galaxy types remains valid is another open question. 

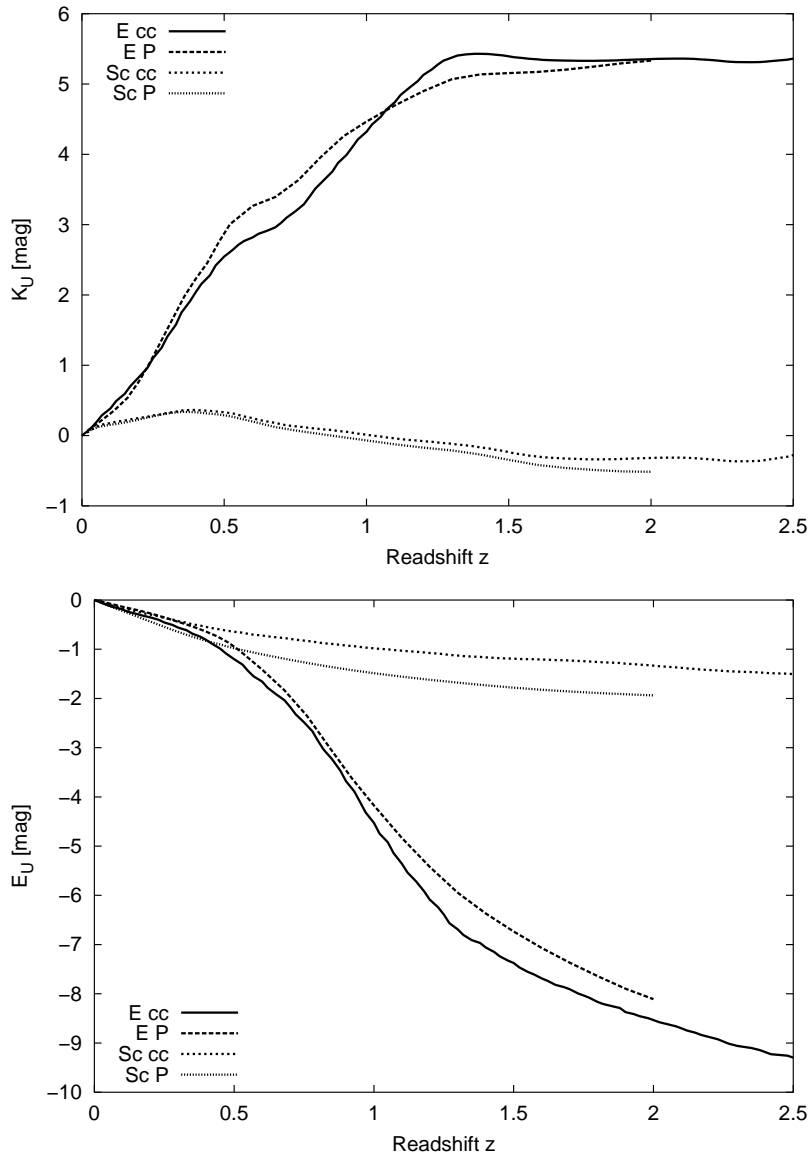

Fig. 10. Comparison between our cc models and the solar models of Poggianti (1997) (P). The top panel shows the $k$-corrections and the bottom panel the $e$-corrections for $\mathrm{E}$ and Sc models in $U$. Note that we compare none-attenuated models in Poggianti's cosmology $\left(H_{0}, \Omega_{0}\right)=$ $(50,0.5)$.

The $\mathrm{E}$ model becomes apparently fainter at redshifts $0<z \lesssim 1(m 450 \sim 24 \mathrm{mag})$, it then starts getting brighter again as it approaches its active star formation phase. At $z \sim 3$ the attenuation comes into play and makes the model get fainter rapidly. By $z=5$ the difference between the attenuated and the non-attenuated models increases to $\sim 10 \mathrm{mag}$.

The $\mathrm{Sa}$ and $\mathrm{Sd}$ models become fainter at redshifts $0<z \lesssim 1$, with $m 450 \sim 24$ mag for Sa and $m 450 \sim 27$ mag for $\mathrm{Sd}$ at $z \sim 1$. Thenceforward both spiral models get moderately fainter until the attenuation comes into play at $z \sim 3$.

In comparison to the HDF data from Sawicki et al. (1997), the Sa model follows the bright limit familiar well. The Sd model gets fainter than the observational limit of $m 450 \lesssim 29$ mag beyond $z=2$ while the classical initial collapse $\mathrm{E}$ model is too bright at $z \gtrsim 2$ to be comparable with the data. The lack of luminous galaxies at redshifts lower than 0.5 is the selection effect explicitly intended by the choice of the HDF. The lack of galaxies as luminous as our classical E model, however is real and not due to any selection or bias. But on the basis of the luminosity we can not determine the role of attenuation. To do this observations $z \gtrsim 4.5$ are needed.

More information is provided by the colours of galaxies. For the $(m 450-m 606)$ colour evolution it is seen that for
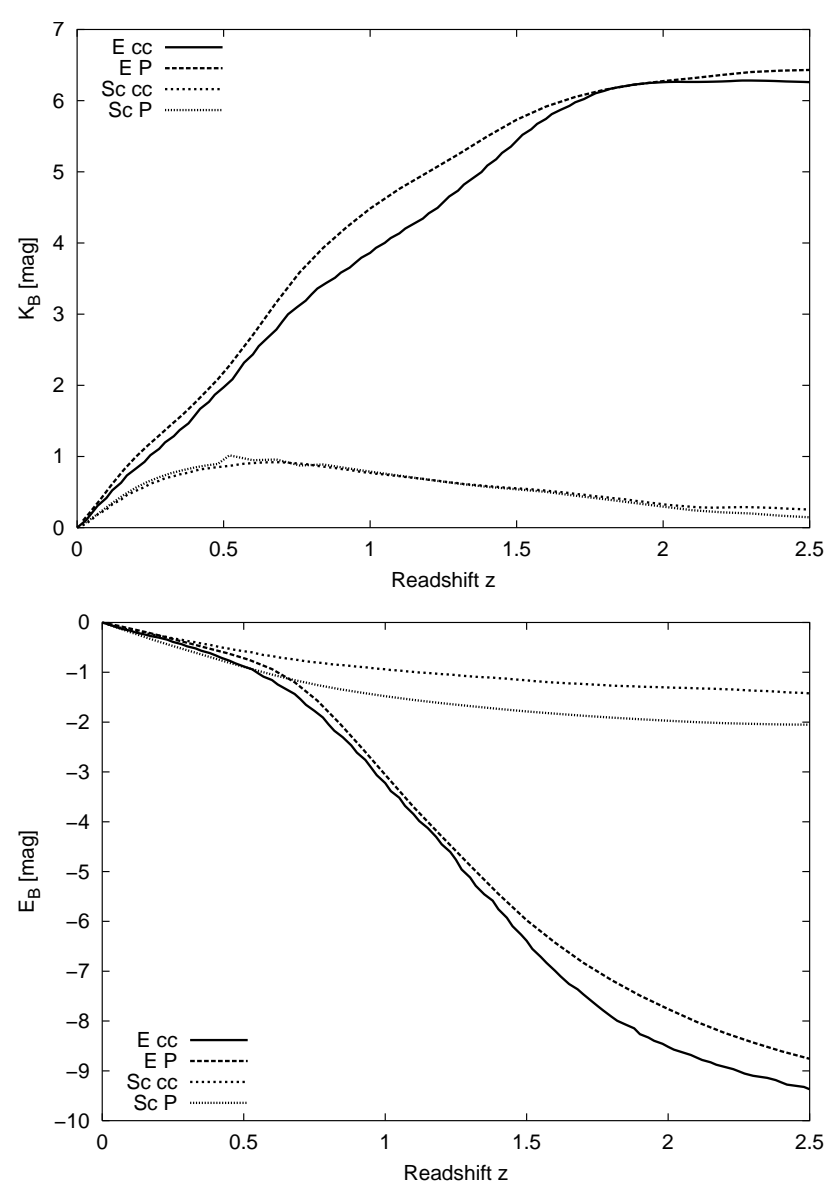

Fig. 11. Same like Fig. 10, but now in the $B$-band.

redshifts $z \geq 2$ the E model gets as blue as the spirals. This is partly due to the strong light contributions from low metallicity stars and partly due to the youth of its stellar population (age $\sim 1.6 \mathrm{Gyr}$ at $z \sim 2$ ). In the redshift range $0.25 \lesssim z \lesssim 0.75$ the $\mathrm{E}$ model is very red $(m 450-m 606) \sim 1.7$. The colour difference between the $\mathrm{Sa}$ and Sd models is small, $\lesssim 0.3$. The models follow the data well, but there are a lot of very blue objects which cannot be explained by our models of undisturbed galaxy evolution.

At redshifts $z \gtrsim 3$ the models with attenuation fit the data mutch better than models without attenuation. While at $z \lesssim 1$ a large number of faint and blue galaxies are seen in the HDF, bluer and fainter than our late-type spiral models, the bulk of galaxies at $1 \lesssim z \lesssim 3$ are well compatible wit our normal spiral progenitor models.

A detailed modelling and interpretation of these Lyman Break Galaxies (LBGs) is beyond the scope of the present paper. It will be the subject of a forthcoming paper including a much larger number of LBGs both with spectroscopic and photometric redshifts (see Möller et al. 2001b for first and preliminary results). This investigation will use the full multi-colour information available for HDF and other deep field galaxies.

\section{Conclusions}

Our evolutionary synthesis model is now chemically consistent with respect to both the spectrophotometric and the 

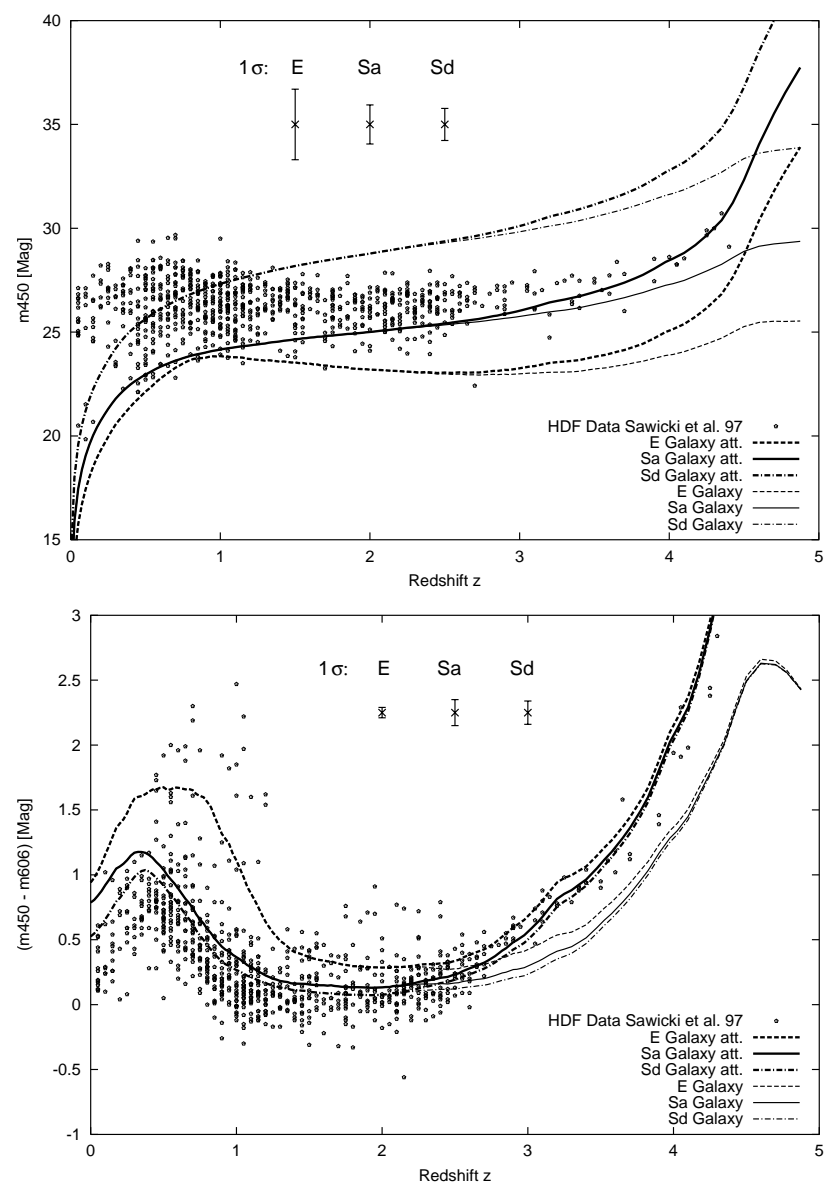

Fig. 12. Redshift evolution of $B$-band luminosity $(m 450)$ and from the $B-V$ colour ( $m 450-m 606$ ) compared to the HDF galaxies photometric redshift catalogue of Sawicki et al. (1997). Vertical bars show the $1 \sigma$ luminosity and colour ranges of local galaxies.

chemical evolution. This gives the possibility for a detailed study of the age and metallicity distributions in the composite stellar populations of galaxies. In the framework of simplified closed-box models where the different spectral types of galaxies $\mathrm{E}, \mathrm{Sa}, \mathrm{Sb}, \mathrm{Sc}$, and Sd are described by their respective appropriate star formation histories we analyse how the presently observed average stellar abundances and abundance distributions have evolved in time.

While - with somewhat different star formation histories - models using input physics for one single metallicity (e.g. solar) only can also be brought into agreement with locally observed galaxy colours and spectra, the evolution with redshift is significantly different.

Before analysing galaxy data out to high redshifts we made sure that our chemically consistent models correctly describe the integrated properties of galaxy types E, Sa-Sd in their spectrophotometric appearance from the UV through the NIR, their average ISM abundances, the metallicity of their stellar populations, their gas content, present-day star formation rates and the chemical abundances of various elements.

This large number of observables allows to tightly constrain the only free parameter of our closed-box 1-zone models: the time evolution of their star formation rate or their star formation history. We show how the stellar metallicity distribution in various galaxy types build up with time to yield after $\sim 12$ Gyr agreement with stellar metallicity distributions observed in our and other local galaxies. We also presented the time evolution of the luminosity-weighted mean stellar metallicities of different galaxy types in various bands. It is these luminosity-weighted metallicities that are expected to be measured by metal-sensitive absorption features observed in the integrated light in the respective passbands.

For the spectral galaxy types E, Sa to Sd, we give the spectral energy distributions over a wide wavelength range (9.09$160000) \mathrm{nm}$ in their time evolution from ages of $1 \mathrm{Gyr}$ up to 15 Gyr.

Any desired set of filter response functions can directly be used to calculate from these spectra the time and redshift evolution of galaxy luminosities, colours, evolutionary and cosmological corrections and apparent magnitudes (for any desired cosmological model).

In comparison with models using solar metallicity input physics only, we discuss the effects of the inclusion of subsolar metallicity stellar subpopulations. In chemically consistent models E galaxies appear significantly fainter to redshifts $z \sim 2.5$ as compared to solar metallicity models. A smaller number of ellipticals is thus expected to contribute to magnitude limited samples.

Chemically consistent spiral models, on the other hand, appear brighter at $z \gtrsim 2$ than they would if only solar metallicity input physics were used. Hence, we expect a larger number of intermediate and late-type spirals from $z \gtrsim 2$ to show up in magnitude limited surveys.

We present a large grid of evolutionary and cosmological corrections as well as apparent magnitudes and colours in various filter systems (Johnson, HST, ...) from UV to NIR including the effect of attenuation by intergalactic $\mathrm{HI}$ for galaxy types $\mathrm{E}$ and $\mathrm{Sa}$ to $\mathrm{Sd}$ using cosmological parameters $\left(H_{0}, \Omega_{0}\right)=(65,0.1)$ with a redshift of galaxy formation assumed to be $z_{\mathrm{f}}=5$.

Models and results our chemically consistent chemical evolution models in terms of a large number of individual element abundances in the ISM of various spiral types were presented in Lindner et al. (1999) and used for the interpretation of Damped Ly $\alpha$ Absorbers.

A very first and preliminary comparison with the redshift evolution of HDF galaxies with photometric redshifts from Sawicki et al. (1997) indicates that their luminosities, colours and SFRs are well compatible with those of our normal spiral models over the redshift interval from $z \sim 0.5$ all through $z>4$.

A detailed and extensive comparison of our chemically consistent model results with the full set of observed colours and luminosities of high redshift galaxies (e.g. Lyman Break Galaxies) will be presented in a forthcoming paper.

In the framework of chemically consistent models it is also possible to include the effects of dust in a largely consistent way, tying the amount of dust to the evolving gas content and metallicity. Stellar track based chemically consistent models with dust will be presented in a companion paper by Möller et al. (in prep., see Möller et al. 2001a,c for first results). 
Acknowledgements. This work was partly supported by the Deutsche Forschungsgemeinschaft (DFG) grant Fr 916/10-1. We thank P. Madau for providing us with the attenuation functions and T. Lejeune for sending us the corrected version of the stellar atmosphere models.

We thank our referee, Dr. L. Carigi, for a insightful and constructive report.

\section{Appendix A: Available data}

The following data are available at our homepage http://www . uni-sw.gwdg.de/ galev/ccmodels/: $e$ - and $k$-corrections, apparent magnitudes, spectra as function of redshift, and spectra as function of evolution time given in restframe. These data are available for all Models (E-Sd). Please see the README file for detailed information and file format.

\section{References}

Aaronson, M. 1978, ApJ, 221, L103

Anders, P., \& Fritze-v. Alvensleben, U. 2003, A\&A, 401, 1063

Arimoto, N., \& Yoshii, Y. 1986, A\&A, 164, 260

Bertelli, G., Bressan, A., Chiosi, C., Fagotto, F., \& Nasi, E. 1994, A\&AS, 106, 275

Bessel, H. L., \& Brett, J. M. 1988, PASP, 100, 1134

Bressan, A., Chiosi, C., \& Fagotto, F. 1994, ApJS, 94, 63

Bruzual, G. A., \& Charlot, S. 1993, ApJ, 405, 538

Buta, R., Mitra, S., de Vaucouleurs, G., \& Corwin, H. G. Jr. 1995, AJ, 107,118

Carigi, L., Colín, P., \& Peimbert, M. 1999, ApJ, 514, 787

Carollo, C. M., \& Danziger, I. J. 1994, MNRAS, 270, $523+743$

Chabrier, G., \& Baraffe, I. 1997, A\&A, 327, 1039

Coleman, G. D., Wu, C. C., \& Weedman, D. W. 1980, ApJS, 43, 393

Einsel, C., Fritze-v. Alvensleben, U., Krüger, H., \& Fricke, K. J. 1985, A\&A, 296, 347

Ferguson, A. M. N., Gallagher, J. S., \& Wyse, R. F. G. 1998, AJ, 116, 673

Ferguson, H. C., Dickinson, M., \& Williams, R. 2000, ARA\&A, 38, 667

Fioc, M., \& Rocca-Volmerange, B. 1997, A\&A, 326, 950

Fioc, M., \& Rocca-Volmerange, B. 1999, A\&A, 351, 869

Fritze-v. Alvensleben, U., \& Gerhard, O. E. 1997, A\&A, 285, 751

Gardner, J. P., Baum, S. A., Brown, T. M., et al. 2000, AJ, 119, 486

Guiderdoni, B., \& Rocca-Volmerange, B. 1987, A\&A, 186, 1

Guiderdoni, B., \& Rocca-Volmerange, B. 1988, A\&AS, 74, 185

van den Hoek, L. B., \& Groenewegen, M. A. T. 1997, A\&AS, 123, 305

Hu, E. M., McMahon, R. G., \& Cowie, L. L. 1999, ApJ, 522, L9

Kennicutt (Jr.), R. C. 1992, ApJS, 79, 255

Kennicutt (Jr.), R. C. 1998, ARA\&A, 36, 189

Kilian-Montenbruck, J., Gehren, T., \& Nissen, P. E. 1994, A\&A, 291, 757

Kroupa, P., Tout, C. A., \& Gilmore, G. 1993, MNRAS, 262, 545

Kurucz, R. L. 1992, in The Stellar Populations of Galaxies, IAU Symp., 149, ed. B. Barbury, \& A. Renzini (Dordrecht: Kluwer), 225

Lamla, E. 1982, in Landolt-Börnstein, ed. K.-H. Hellwege (Springer), Band VI/2b, 73
Lejeune, T., Cuisinier, F., \& Buser, R. 1997, A\&AS, 125, 229

Lejeune, T., Cuisinier, F., \& Buser, R. 1998, A\&AS, 130, 65

Lindner, U., Fritze-v. Alvensleben, U., \& Fricke, K. J. 1996, A\&A, 316,123

Lindner, U., Fritze-v. Alvensleben, U., \& Fricke, K. J. 1999, A\&A, 341, 709

Loewenstein, M. 1999, in Star Formation in Early-Type Galaxies, ed. P. Carral, \& J. Cepa, ASP Conf. Ser., in press

Lowenthal, J. D., Koo, D. C., Guzman, R., et al. 1997, ApJ, 481, 673

Madau, P. 1995, ApJ, 441, 18

McWilliam, A., \& Rich, R. M. 1994, ApJS, 91, 749

Möller, C. S., Fritze-v. Alvensleben, U., \& Fricke, K. J. 1996, in From Stars to Galaxies, ed. C. Leitherer, U. Fritze-v. Alvensleben, \& J. Huchra, ASP Conf. Ser., 98, 496

Möller, C. S., Fritze-v. Alvensleben, U., \& Fricke, K. J. 1997, A\&A, 317,676

Möller, C. S., Fritze-v. Alvensleben, U., \& Fricke, K. J. 1999, in The Birth of Galaxies, Proc. of the Xth Rencontres de Blois, ed. B Guiderdoni, et al. (Éditions Frontières)

Möller, C. S., Fritze-v. Alvensleben, U., Calzetti, D., \& Fricke, K. J. 2001a, IAU Symp., 204, 413

Möller, C. S., Fritze-v. Alvensleben, U., \& Calzetti, D. 2001b, Ap\&SS, 277, 601

Möller, C. S., Fritze-V. Alvensleben, U., Fricke, K. J., \& Calzetti, D. 2001c, Ap\&SS, 276, 799

Oey, M. S., \& Kennicutt, R. C. Jr. 1993, ApJ, 411, 137

Pettini, M., King, D. L., Smith, L. J., \& Hunstead, R. W. 1997, ApJ, 510, 576

Pettini, M., Ellison, S. L., Steidel, C. C., \& Bowen, D. V. 1999, ApJ, 510,576

Pettini, M., Steidel, C. C., Adelberger, K. L., Dickinson, M., \& Giavalisco, M. 2000, ApJ, 528, 96

Phillipps, S., \& Edmunds, M. G. 1996, MN, 281, 362

Poggianti, B. M. 1997, A\&AS, 122, 399

Ramirez, C., Solange, V., Stephens, et al. 2000, AJ, 120, 633

Richer, M. G., \& McCall, M. 1995, ApJ, 445, 642

Rocha-Pinto, H. J., \& Maciel, W. J. 1998, A\&A, 339, 791

Sadler, E. M., Rich, R. M., \& Terndrup, D. M. 1996, AJ, 112, 171

Sandage, A., Binggeli, B., \& Tamman, G. A. 1985a, AJ, 90, 395

Sandage, A., Binggeli, B., \& Tamman, G. A. 1985b, AJ, 90, 1759

Sawicki, M., \& Yee, H. K. C. 1997, AJ, 113, 1

Scalo, J. M. 1986, Fundamentals of Cosmic Physics, 11, 1

Schulz, J., Fritze-v. Alvensleben, U., Möller, C. S., \& Fricke, K. J. 2002, A\&A, 392, 1

Teplitz, H. I., Malkan, M. A., Steidel, C. C., et al. 2000, ApJ, 542, 18

Trager, S. C., Faber, S. M., Dressler, A., \& Oemler, A. 1997, ApJ, 485, 92

Vazdekis, A., Casuso, E., Peletier, R. F., \& Beckman, J. E. 1996, ApJS, 106, 307

Vazdekis, A., Peletier, R. F., Beckman, J. E., \& Casuso, E. 1997, ApJS, 111,203

Vázquez, G. A., Carigi, L., \& González, J. J. 2003, A\&A, 400, 31

Williams, R. E., Blacker, B., Dickinson, M., et al. 1996, AJ, 112, 1335

Williams, R. E., Baum, S. A., Bergeron, L. E. et al. 1998, Am. Astron. Soc. Meet., 193, 7501

Zaritsky, D., Kennicutt, R. C., \& Huchra, J. P. 1994, ApJ, 420, 87

van Zee, L., Salzer, J. J., Haynes, M. P., et al. 1998, AJ, 116, 2805 